\title{
Multiphase fault tolerant distributed control techniques for integrated drives based on resonant regulators
}

\author{
Filippo Savi*, Davide Barater ${ }^{\dagger}$, Giampaolo Buticchi ${ }^{* \ddagger}$, Pat Wheeler ${ }^{* \ddagger}$, Chris Gerada $* \ddagger$ \\ ${ }^{*}$ Zhejiang Key Laboratory on the More Electric Aircraft Technologies, \\ University of Nottingham Ningbo China, Ningbo 315100, China (e-mail: buticchi @ieee.org) \\ ${ }^{\dagger}$ Department of Engineering Enzo Ferrari, \\ University of Modena and Reggio Emilia, Modena 41125, Italy (e-mail: davide.barater@unimore.it)

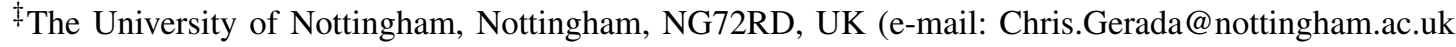

\begin{abstract}
One of the challenges brought forward by the gradual electrification undertaken by the aviation sector is the requirement of fault tolerance for machine drive systems to be used for critical on-board tasks such as propulsion or primary flight surface actuation. Their inherent advantages in both volumetric and gravimetric power density makes integrated drives the prime candidates for these applications. Despite the large advances in this field, few key area still need work. Key among which is fault tolerant current control strategies. This paper studies the application of resonant control techniques to achieve a scalable and fault tolerant current control strategy for multiphase machine.
\end{abstract}

\section{INTRODUCTION}

In the field of aircraft design and manufacturing, several non critical systems have been moved from pneumatic, mechanical or hydraulic power to electrical, thanks to the flexibility and conversion efficiency that this form or energy inherently has. This, embodied in the More Electric Aircraft (MEA) movement has already led to improved fuel efficiency with all the consequent environmental and economic benefits that it entails in the last airliners introduced to market by all manufacturers. One area where large gains are still expected to be possible is in the electrification of the more critical systems such as primary flight surface controls [1] or propulsion. The ability to effectively decouple power and thrust generation is predicted to play a large role in the future aircraft designs, as shown in [2], [3]. Several challenges need to be solved in order to achieve the stated vision. A demand for a ever larger power density, to allow the use of electric actuation in the space and weight constrained on board environment, pushes toward the adoption of integrated drives; eliminating bulky and heavy cables and separate electronics enclosures. Another issue that still requires more research in this space is the relative lack of fault tolerance of the actuation architectures commonly used today. The standard three phase machine, while technically capable of operation under some specific fault conditions, if coupled with a suitable control mechanism, as shown in [4], shows significant performance degradation, with very large torque fluctuations, in addition to the loss of at least a third of the maximum available power (additional de-rating might be necessary for thermal reasons). The commonly proposed solution to address this issue is the adoption of multi-phase or multi three phase machines, where the loss of one or more windings has much less impact on the overall system, as summarized in [5].

On the power electronics front, fault tolerance poses a sensibly larger challenge, as failures, especially when under heavy load, which is in fact the most likely scenario, are much more difficult to deal with. The transient subsequent these events will impose large voltage and current stress on both the power devices themselves, the gate drive electronics and logic behind them, as the energy is redistributed throughout the system. In these instances parasitic coupling, which would be normally negligible can transfer significant amount of energy to circuits not able to handle it; due to the large voltage and current temporal gradients. This leads to signal integrity caused secondary failures that while just as damaging, can be even more difficult to successfully deal with.

One possible way to address this issue is to distribute the power electronics, separating the usually completely integrated drives, in several independent cells, each one of them responsible to control a single phase. This eliminates the parasitic elements through physical and electrical separation, decreasing the chance of a secondary fault [6].

On the machine control front, fault tolerance poses a challenge for all the most used control strategies, as the number of phases in a machine and the relative relationship between them are usually assumed as constants. In Field Oriented Control (FOC) for example, both set-points and measurements are mapped through specific transformations to a different vector space, the so called rotating reference frame, this allows the control to be performed on quasi-static signals. Unfortunately since there is no one to one correspondence between signals in the two domains. It is necessary to use a different transforms for each configuration of the machine, as in [7]. It is possible to make analogous considerations for Direct Torque Control (DTC), where the available voltage states change with the machine configurations, as shown in [8]. Both these approaches do not scale well with an increasing 


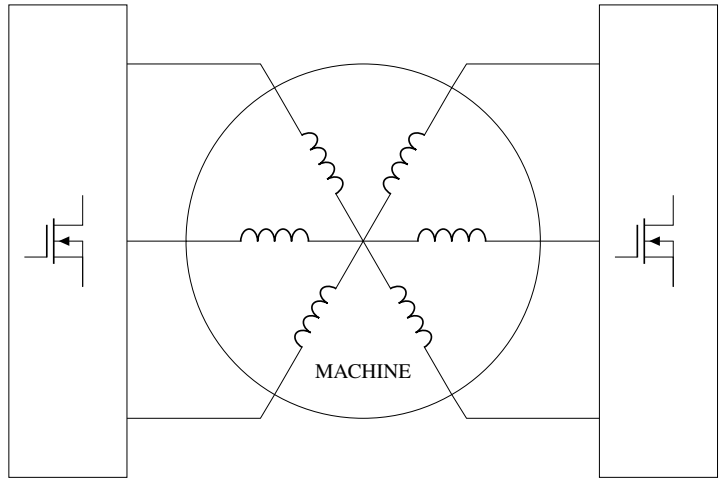

Fig. 1. Traditional fault tolerant architecture

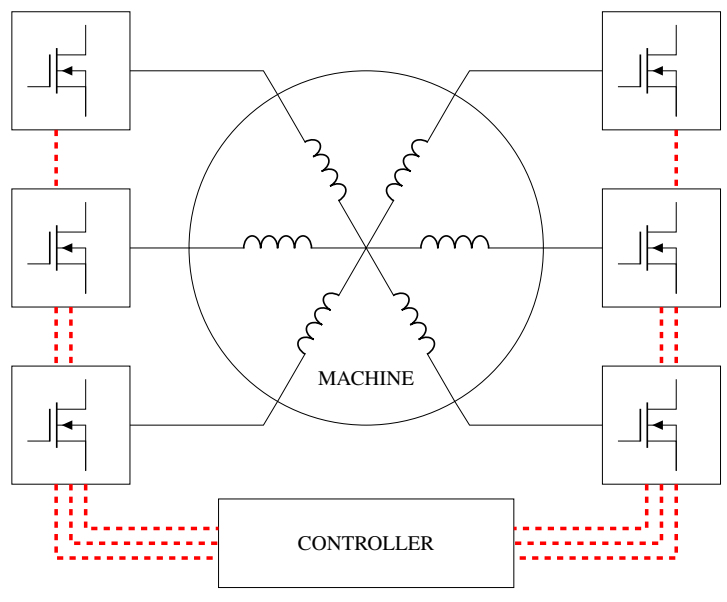

Fig. 2. Proposed fault tolerant architecture

phase count, as the number of different machines configuration grows exponentially.

This paper introduces a different control architecture, where the stator current control is performed directly in the static reference frame, making it completely independent from machine structure, as each phase is controlled independently. This not only makes it trivial to transition between machine configurations by just isolating defective phases, but is greatly simplifies control in machines with an high degree of magnetic coupling between phases, as each controller acts on a single phase and not on a linear combination of multiple ones.

To achieve the previously stated goal, a controller that is capable of tracking sinusoidal signals is needed, this excludes the commonly used PI and PID regulators. Proportional resonant controllers (PR) are thus used in their place. These type of controllers are already extensively used for fixed frequency current control in grid tied inverters [9], [10], and have been proposed for use in specific circumstances for speed control in machine drives [11], [12]. This paper aims to extend their use to current control for variable frequency drives.

In chapter 2 the overall system architecture and proposed control scheme is detailed, chapter 3 presents how the system has been modeled. The $H_{\infty}$ stability analysis is presented in chapter 4, last but not least Simulations are shown in chapter
TABLE I

SYSTEM PARAMETERS

\begin{tabular}{lc}
\hline Parameter name & Value \\
\hline Machine maximum Torque & $10 \mathrm{~N} \mathrm{~m}$ \\
Machine maximum speed & $10000 \mathrm{rpm}$ \\
Machine rated current & $50 \mathrm{~A}$ \\
Converter maximum output voltage & $300 \mathrm{~V}$ \\
Converter maximum output current & $180 \mathrm{~A}$ \\
\hline
\end{tabular}

5.

\section{SYSTEM ARCHITECTURE}

\section{A. Hardware}

In Figure 2, the architecture for the proposed system is shown, as opposed to a more traditional fault tolerant topology with two regular three phase drives in 1 . First and foremost we have the machine, represented in the diagram as star connected, six phase, with floating neutral point and symmetrical windings. While other fault tolerant configurations are possible, namely the multi three phase with separate sets of windings, the differences, with respect to the proposed control strategy, are minimal. The proposed strategy is also able to manage an arbitrary number of phases, having been designed specifically not to have any scalability bottleneck. For this reason only this configuration will be taken into consideration throughout the rest of this paper.

The power electronics hardware consists of six separate single phase cells, each one controlling a single phase. These are all connected to a centralised control structure through a custom, point to point, low latency, power electronics digital communication protocol [6]. While this design decision, on the surface, introduces a single point of failure, in the controller. It allows the system to achieve higher performance and better flexibility when compared to fully distributed droop based control architectures. Moreover this flaw can be addressed and completely eliminated, through the use of well known redundancy techniques at the communication level, like triple modular redundancy [13]. It should also be noted that logic circuits are much less stressed as opposed to the power semiconductors, and thus are generally more reliable, according to physics of failure analysis [14].

The other fundamental task carried out by the communication system is the control of cell timing. through the use of either a dedicated clock distribution network or clock recovery techniques. This allows the system to achieve total synchronisation between nodes, coupling this with the use of Field Programmable Gate Array (FPGA) for the low level control implementation with deterministic timing behaviours allow the overall distributed system to act as a single entity. The main parameters for the system are shown in table I

\section{B. Control system}

A comprehensive structure of the proposed controller, is shown in figure 3 , this derived from the classical field oriented 


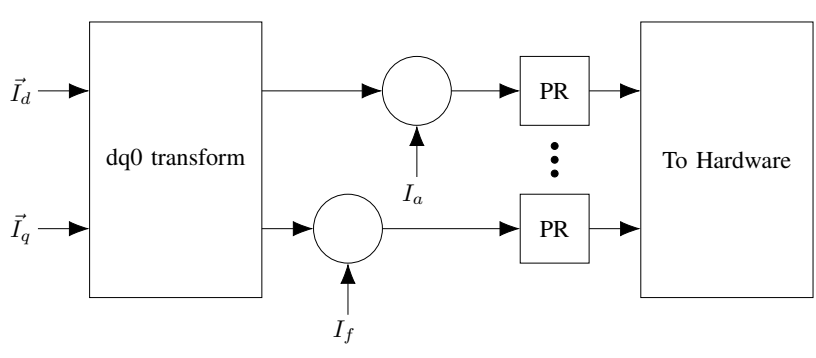

Fig. 3. Proposed control system overview

control scheme. The setpoint, presented as direct and quadrature current vectors in the $d q 0$ space are used to generate a multi-phase set of reference currents, through an appropriate transform, a bank of proportional resonant (PR) controllers tracks this, now sinusoidal reference, generating a voltage setpoint that is then transmitted through the communication infrastructure to each single converter.

This structure grants the overall system a large degree of resiliency from several classes of fault, allowing safe operation to be carried on in almost any situation, as long as a majority of the system is still intact. Communication faults to single modules, due to loose connections or cut cables, can be detected by the local cell controller, will lead to a gradual shutdown of only the affected phase. With the master controller, once notified by the communication fault detection algorithm, only shutting down only the affected PR controller, without, directly affecting any other phase or creating stability problems. A similar chain of events will also take place in case of an hardware fault in one of the phases, which will either be reported to the main controller, if the local cell logic is still functioning regularly, or will present similar symptoms as lost communication otherwise.

The system while able to continue in this state safely for an indefinite amount of time, can also optionally be reconfigured, for example by swapping the transform with a different one, to increase the post-fault performance (decreasing ripple or increasing available power output). This is in stark contrast with the ordinary FOC where transforms and controllers must be reconfigured as quickly as possible to avoid potential stability problems, and consequent risks of hardware damages beyond the original fault.

\section{SyStem MODELING}

The foundation of a solid control strategy is always a simplified mathematical model that capture all the necessary dynamics of the core system, that can then be then extended as needed in order to include higher order effects or other interconnected systems. Some starting assumptions have been made in order to both simplify and limit the scope of the problem:

- the input dc voltage source is considered ideal, while this can be limiting in some scenarios, the characteristics of the power distribution network can vary widely both between installations, and for different load.
- the hardware bandwidth of the converter in itself is much wider than both the load and desired control loop bandwidth. As opposed to the previous one this is easily verified, as the wide band-gap devices and optimised physical layout are capable of supporting current bandwidths well into the Megahertz frequency range.

\section{A. Machine modeling}

The first step in the modelling of the machine is the extraction of a purely electrical model, here the classical approach has been followed, as shown in eq. 1, starting with an RL series circuit modeling the physical characteristics of the armature winding and a ideal AC voltage source of Back EMF that takes care of all interactions with the rotor and load dynamics.

$$
\begin{aligned}
& V_{I N}=R I(s)+s L I(s)+E(s) \\
& E(s)=\frac{P_{M E C H}(s)}{T}=\frac{\eta P_{E L E}(s)}{T}=\frac{\eta V_{I N}(s) I(s)}{T}
\end{aligned}
$$

The last term is then detailed through the use of basic mechanical relationships in order to explicitly show the dependence on the load torque. This model however still presents the problem of not being linear, as it contains the electrical power. To avoid having to resort to non-linear control techniques, this therm has been linearized around a generalised operating point $\left(V_{0}\right.$, $I_{0}$ ) with a first order Taylor expansion, leading to The transfer function shown in 2

$$
P(s)=\frac{I_{O U T}(s)}{V_{I N}(s)}=\frac{1-I_{0} \frac{\eta K e}{T}}{R+s L+V_{0} \frac{\eta K e}{T}}
$$

A second Effect that needs to be evaluated as it heavily influences the machine dynamics is the acceleration torque due to the rotor moment of inertia. To integrate this effect into the previously developed model, the overall machine torque is split into two contributing factors, a mechanical term due to the desired load and inertia term. Again as before the back EMF term must be linearised as now both power and inertia term are non-linear. The resulting model has two inputs (voltage and machine angular speed) and a single output (Current in the winding). and is characterised by the following transfer matrix.

$$
I=\left[\begin{array}{c}
\frac{1-I_{0} \frac{K_{e} \eta}{T_{M E C H}}}{R+s L+V_{0} \frac{K_{e} \eta}{T_{M E C H}}} \\
\frac{J \frac{2}{n p} s}{T_{M E C H}\left(R+s L+\frac{V_{0}}{T_{M E C H}}\right)}
\end{array}\right]\left[\begin{array}{l}
V_{I N} \\
\omega_{e}
\end{array}\right]
$$

Other mechanical effects such as bearing friction and windage are not considered as they play only a minor role in the vast majority of electrical machines, for this reason care should be taken with special machine classes where this assumption does not apply (ultra high speed designs). Another core assumption of the model that is generally considered to be valid for the intended application is for the moment of inertia to be large enough to allow the related contributions to be considered quasi-static for the purposes of considering system dynamics. 


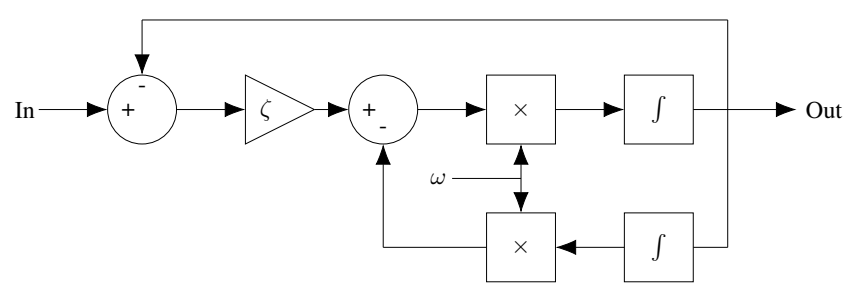

Fig. 4. Block diagram of the second order generalised integrator.

\section{B. Controller modeling}

The PR controller is conceptually very similar to a traditional proportional integral controller, where the simple integrator has been substituted with a Second Order Generalised Integrator (SOGI), shown in figure 4. Its transfer function, shown in 3, allows, depends on the value of the damping term $\zeta$ to get arbitrarily high gain at the desired frequency $\omega^{\prime}$, allowing potentially to have infinite gain when $\zeta=0$. This, while guaranteeing zero steady state error, is often undesirable as it can lead to instability and also poses numerical implementation challenges. a careful tuning of the parameter is ultimately dependant on a trade-off between achievable controller performance, stability and capability of the chosen platform.

$$
Y(s)=\frac{\zeta \omega^{\prime} s}{s^{2}+\zeta \omega^{\prime} s+\omega^{\prime 2}} E(s)
$$

When this type of controller is used in variable frequency applications, the controller has now to be treated as a MIMO system, as both error $E$ and frequency $\omega^{\prime}$ are now inputs. So it is not possible to extract the transfer functions directly from equation 3. To tackle this issue a linearized version 4 , derived in [15] has been used in the following steps.

$$
S O G I(s)=\frac{k \omega_{0} s}{s^{2}+k \omega_{0} s+\omega_{0}^{2}} E(s)-2 \frac{k \omega_{0}^{2}}{s^{2}+k \omega_{0} s+\omega_{0}^{2}} \frac{\omega}{\omega_{0}}
$$

Finally the transfer matrix of the PR controller 5 is determined by scaling proportional and resonant actions with appropriate gains $K_{P}$ and $K_{R}$ and then combining them together.

$$
Z=\left[\begin{array}{c}
\frac{K_{P} * s^{2}+\omega_{0} * \zeta *\left(K_{P}+K_{R}\right) * s+K_{P} * \omega_{0}^{2}}{s^{2}+\zeta * \omega_{0} * s+\omega_{0}^{2}} \\
\frac{-2 * \zeta * \omega_{0}}{s^{2}+\zeta * \omega_{0} * s+\omega_{0}^{2}}
\end{array}\right]\left[\begin{array}{l}
E(S) \\
\Omega(S)
\end{array}\right]
$$

\section{STABILITY}

It is of critical importance to evaluate the stability of a control scheme in order to insure the desired behaviour is safely maintained in all possible situations. To achieve this goal several have been carried out. At first the stability of the target system has been evaluated through conventional methods such as pole/zero analysis and Nyquist criterion. Successively in order to evaluate the system's stability even in presence of noise and disturbances, the structured singular

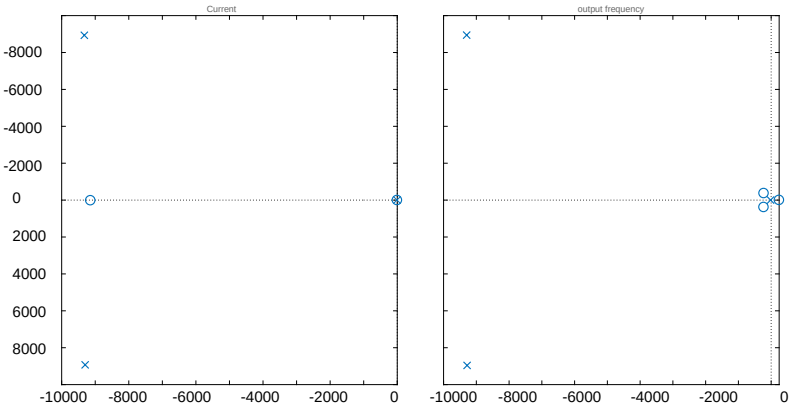

(a) Pole zero map
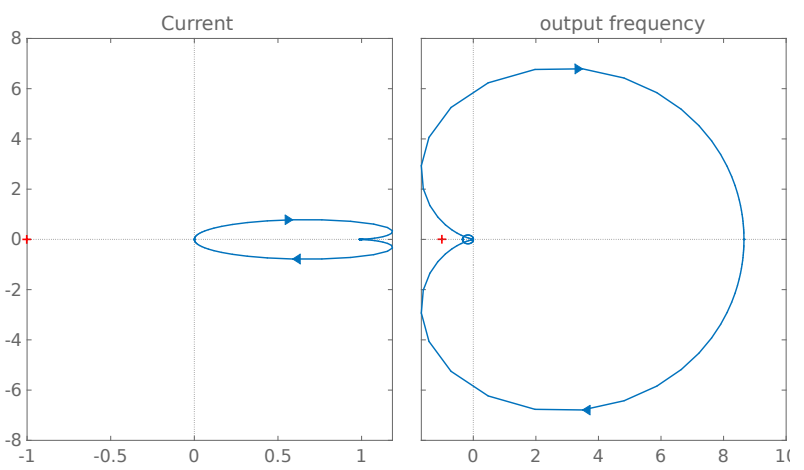

(b) Nyquist plot

Fig. 5. Conventional stability analysis results

value analysis [16], [17] ( $\mu$ analysis) is used, which can quantify the robust stability performance of a system.

The results of the stability analysis are shown in figure 5, both in term of a Nyquist diagram, and a pole zero map. The system has no poles in the right hand side of the plane, thus it can be concluded that the system is stable; it must be also denoted that the poles on the imaginary axis, while usually undesired, are vital to the functions of the control scheme as they allow high gain at the desired frequency allowing the controllers to track sinusoidal references with no steady state error.

The robust stability evaluation has been performed on the same system described in section II-A, with a $50 \%$ uncertainty applied to the machine magnet flux and rotor inertia, as these two values are typically difficult verify experimentally with sufficient precision. The calculated $\mu$ factor of 220 shows extreme robustness of the control algorithm to any change in any of these two factors.

\section{Simulation}

After the theoretical stability analysis the performance of the proposed control have been simulated, using Simulink for the control algorithm, whose parameters are shown in table $\mathrm{V}$ and PLECS, a piece-wise linear simulator, for the electronics and machine.

After the initial start up and acceleration transient the machine is run at a constant speed through a simple PI control loop. At 10 millisecond a load torque step is applied. 
TABLE II

CONTROLLER PARAMETERS

\begin{tabular}{ll}
\hline Parameter name & Value \\
\hline $\mathrm{Kp}$ & 10 \\
$\mathrm{Kr}$ & 35 \\
$\mathrm{Z}$ & 0.8 \\
\hline
\end{tabular}

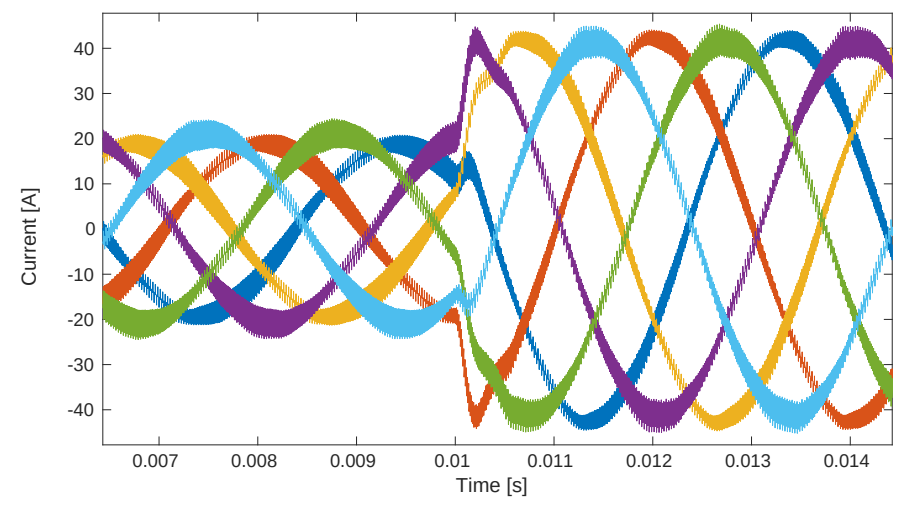

Fig. 6. Simulated Load Step

In figure 6 the stator currents during this test are shown. The highlighted behaviour of the control loops is in line with expectations with fast response and minimal overshoot. While this test demonstrates the good behaviour of the proposed control in case of a healthy system, it's behaviour in a fault scenario still needs to be validated. Since a comprehensive analysis of every possible fault scenario is not possible, due to the very large number of possibilities that would entail; one of the worst case scenarios, from the point of view of the current control has been examined. In particular an open circuit fault in the machine is considered. To emulate this scenario, after the start-up transient is finished, a high value resistor is inserted in series to one of the phases, in order to prevent any current flow. Simulating a broken connection either within the machine itself or in its connection to the drives.

The results of this last simulation, in figure $\mathrm{V}$, show that

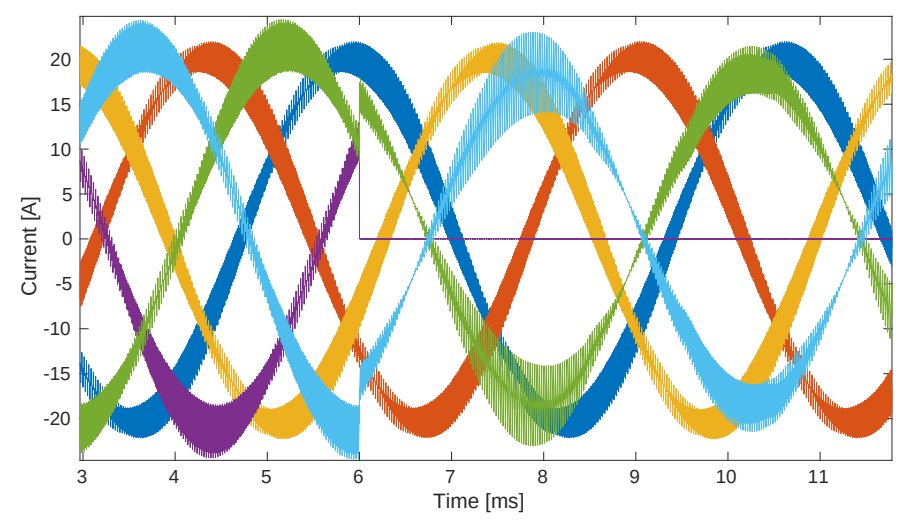

Fig. 7. Simulated Phase loss the control strategy is able to maintain control of the currents both through and after the fault, allowing safe operation of the system, albeit with slightly degraded performances

\section{CONCLUSIONS}

In this paper a highly scalable fault tolerant control architecture it is presented, that allows the control any type of multiphase electrical machine. Thanks to the resonant controllers stability and full control authority can be maintained throughout the fault event on the healthy phases and winding, as demonstrated through simulations. The theoretical stability of the system has also been evaluated through both conventional and robust techniques.

\section{REFERENCES}

[1] W. Lee, S. Li, D. Han, B. Sarlioglu, T. A. Minav, and M. Pietola. A review of integrated motor drive and wide-bandgap power electronics for high-performance electro-hydrostatic actuators. IEEE Transactions on Transportation Electrification, 4(3):684-693, 2018.

[2] V. Madonna, P. Giangrande, and M. Galea. Electrical power generation in aircraft: Review, challenges, and opportunities. IEEE Transactions on Transportation Electrification, 4(3):646-659, 2018.

[3] G. Buticchi, S. Bozhko, M. Liserre, P. Wheeler, and K. Al-Haddad. On-board microgrids for the more electric aircraft-technology review. IEEE Transactions on Industrial Electronics, 66(7):5588-5599, 2019.

[4] A. Kontarček, P. Bajec, M. Nemec, V. Ambrožič, and D. Nedeljković. Cost-effective three-phase pmsm drive tolerant to open-phase fault. IEEE Transactions on Industrial Electronics, 62(11):6708-6718, 2015.

[5] W. Cao, B. C. Mecrow, G. J. Atkinson, J. W. Bennett, and D. J. Atkinson. Overview of electric motor technologies used for more electric aircraft (mea). IEEE Transactions on Industrial Electronics, 59(9):3523-3531, 2012.

[6] Valentina Bianchi, Filippo Savi, Ilaria De Munari, Davide Barater, Giampaolo Buticchi, and Giovanni Franceschini. Minimization of network induced jitter impact on fpga-based control systems for power electronics through forward error correction. Electronics, 9(2):281, Feb 2020.

[7] H. Zhou, W. Zhao, G. Liu, R. Cheng, and Y. Xie. Remedial field-oriented control of five-phase fault-tolerant permanent-magnet motor by using reduced-order transformation matrices. IEEE Transactions on Industrial Electronics, 64(1):169-178, 2017.

[8] M. Bermudez, I. Gonzalez-Prieto, F. Barrero, H. Guzman, M. J. Duran, and X. Kestelyn. Open-phase fault-tolerant direct torque control technique for five-phase induction motor drives. IEEE Transactions on Industrial Electronics, 64(2):902-911, 2017.

[9] H. Komurcugil, N. Altin, S. Ozdemir, and I. Sefa. Lyapunov-function and proportional-resonant-based control strategy for single-phase gridconnected vsi with lcl filter. IEEE Transactions on Industrial Electronics, 63(5):2838-2849, May 2016.

[10] A. Timbus, M. Liserre, R. Teodorescu, P. Rodriguez, and F. Blaabjerg. Evaluation of current controllers for distributed power generation systems. IEEE Transactions on Power Electronics, 24(3):654-664, March 2009.

[11] G. Ellis and R. D. Lorenz. Resonant load control methods for industrial servo drives. In Conference Record of the 2000 IEEE Industry Applications Conference. Thirty-Fifth IAS Annual Meeting and World Conference on Industrial Applications of Electrical Energy (Cat. No.00CH37129), volume 3, pages 1438-1445 vol.3, 2000.

[12] C. Xia, B. Ji, and Y. Yan. Smooth speed control for lowspeed high-torque permanent-magnet synchronous motor using proportional-integral-resonant controller. IEEE Transactions on Industrial Electronics, 62(4):2123-2134, 2015.

[13] T. Arifeen, A. S. Hassan, and J. Lee. Approximate triple modular redundancy: A survey. IEEE Access, 8:139851-139867, 2020.

[14] M. Galea, P. Giangrande, V. Madonna, and G. Buticchi. Insulation systems for electrical machines: Reliability must become a main design objective. IEEE Industrial Electronics Magazine, 2019.

[15] H. Yi, X. Wang, F. Blaabjerg, and F. Zhuo. Impedance analysis of sogifll-based grid synchronization. IEEE Transactions on Power Electronics, 32(10):7409-7413, Oct 2017. 
[16] K. Zhou and J. C. Doyle. Essentials of Robust Control. Prentice-Hall, 1998.

[17] R. Rosso, J. Cassoli, G. Buticchi, S. Engelken, and M. Liserre. Robust stability analysis of lcl filter based synchronverter under different grid conditions. IEEE Transactions on Power Electronics, 34(6):5842-5853, 2019. 